\title{
Global Attractor for Caginalp Hyperbolic Field-phase System with Singular Potential
}

\author{
Daniel Moukoko $^{1}$, Fidèle Moukamba ${ }^{1}$ \& Langa Franck Davhys Reval ${ }^{1}$ \\ ${ }^{1}$ Faculté des Sciences et Techniques, Université Marien Ngouabi, Brazzaville BP 69, Congo \\ Correspondence: Fidèle Moukamba, Faculté des Sciences et Techniques, Université Marien Ngouabi, Brazzaville \\ BP 69, Congo. E-mail: moukambaf@yahoo.fr
}

Received: May 8, 2015 Accepted: May 25, 2015 Online Published: August 7, 2015

doi:10.5539/jmr.v7n3p165 URL: http://dx.doi.org/10.5539/jmr.v7n3p165

\section{Abstract}

This article is devoted to the study of the Caginalp hyperbolic phase-field system with singular potentials. We first prove the existence and uniqueness of solutions for Caginalp hyperbolic phase-field system with logarithmic potential. We then prove the existence of global attractor. One of main difficulties is to prove that the solutions are strictly separated from singular values of the potential.

Keywords: Caginalp hyperbolic phase-field system, logarithmic potential, Dirichlet boundary conditions, phase space, bounded absorbing set, dissipativity, global attractor

Subject Classification: Primary: 35D05, 35D10, 35D20, 35D25,35D30, Secondary: 46F30

\section{Introduction}

The global attractor is a compact invariant and smallest set which attracts the bounded sets of phase space. It presents two major default: it can attract the trajectories slowly and it can be sensitive to perturbations. In this article, we are interested in the study of the following Caginalp hyperbolic phase-field system in a smooth and bounded domain $\Omega \subset \mathbb{R}^{n}, 1 \leq n \leq 3$,

$$
\begin{aligned}
\epsilon \partial_{t}^{2} u+\partial_{t} u-\Delta u+f(u) & =\partial_{t} \alpha \\
\partial_{t}^{2} \alpha-\partial_{t} \Delta \alpha-\Delta \alpha & =-\partial_{t} u
\end{aligned}
$$

with homogenous Dirichlet conditions

$$
u=\alpha=0 \text { on } \partial \Omega,
$$

and initial conditions

$$
\left.u\right|_{t=0}=\left.u_{0} \quad \partial_{t} u\right|_{t=0}=\left.u_{1} \quad \alpha\right|_{t=0}=\left.\alpha_{0} \quad \partial_{t} \alpha\right|_{t=0}=\alpha_{1},
$$

where $\epsilon>0$ is a relaxation parameter, $u=u(t, x)$, the order parameter and $\alpha=\alpha(t, x)$ are unknown functions, $f$ is a given singular potential function.

Consider the following logarithmic potential function

$$
f(s)=-k_{0} s+k_{1} \ln \frac{1+s}{1-s}, \quad s \in(-1,1), 0<k_{1}<k_{0} .
$$

The function $f$ satisfies the following properties

$$
\begin{aligned}
& f \in C^{2}(-1,1), \quad \lim _{s \rightarrow \pm 1} f(s)= \pm \infty, \quad \lim _{s \rightarrow \pm 1} f^{\prime}(s)=+\infty \\
& -c_{0} \leq F(s) \leq f(s) s+c_{0} \text { where } F(s)=\int_{0}^{s} f(\tau) \mathrm{d} \tau \text { and } c_{0}>0, \\
& -c_{1} \leq f^{\prime}(s), \quad c_{1}>0 \quad \forall s \in[0,1) .
\end{aligned}
$$

The system (1) - (2) has a sense only if

$$
-1<u(t, x)<1
$$

for almost all $(t, x) \in \mathbb{R}_{+} \times \Omega$. This reason leads us to introduce the following quantity

$$
\mathbb{D}[u(t)]=\left(1-\|u(t)\|_{L^{\infty}}\right)^{-1} .
$$


We introduce also the standard energy norm of the initial and boundary value problem for singularly perturbed damped hyperbolic equation (1)

$$
\left\|\zeta_{u}\right\|_{\mathcal{E}^{k}(\epsilon)}^{2}=\|u\|_{H^{k+1}}^{2}+\epsilon\left\|\partial_{t} u\right\|_{H^{k}}^{2}+\left\|\partial_{t} u\right\|_{H^{k-1}}^{2},
$$

where $\varepsilon^{\kappa}(\epsilon)$ coincides with $\left[H^{\kappa+1}(\Omega) \times H^{\kappa}(\Omega)\right] \cap\left\{\left.\zeta\right|_{\partial \Omega}=0\right\}$. This standard energy norm is used in (Fabrie, Galusinski, Miranville \& Zelik, 2014), (Grasselli, Miranville, Pata \& Zelik, 2007), ( Moukoko 2014, 2015).

The Caginalp parabolic system, with various types of boundary conditions and for a regular or singular potentials $f$, has been extensively studied, ( see, e.g.,(Cherfils, Gatti \& Miranville, 2008), (Conti, Gatti \& Miranville, 2012), (Cherfils \& Miranville, 2009), (Efendiev, Miranville \& Zelik, 2003 , 2004), (Fabrie, Galusinski, Miranville \& Zelik, 2014), (Miranville \& Quintanilla, 2009). There are few studies concerned the Caginalp parabolic-hyperbolic system.

Recently, above Caginalp hyperbolic phase-field system endowed with homogenous Dirichlet boundary conditions with a regular potentials, is studied in (Moukoko 2014, 2015), in order to prove the existence and uniqueness of solutions, existence of: global attractor, exponential attractors and the robust family of exponential attractors.

Here we are interested in the Caginalp hyperbolic system with homogenous Dirichlet boundary conditions and logarithmic potential. We prove the existence and uniqueness of solutions, as well as regularity. The main difficulties in this article is to prove that the order parameter $u$ is strictly separated from the singular values of the potential.

In this article, we denote by $\|$.$\| and (., .) ( or \|.\|_{\phi}$ ) the norm and the scalar product in $L^{2}(\Omega)$ ( in $\Phi$ ).

\section{Method}

In this section we brief on method needed to prove our two main results of the next section.

We first prove the existence of the solution which are separated from the singular values \pm 1 of the singular potential $f$. We replace the logarithmic potential by a regular function and prove that the solution of the resulting system is also the solution of initial system. We define two phase spaces. in order to end, we first prove the existence of the bounded absorbing set in each phase space, and owing to (Miranville \& Zelik, 2008), we prove the existence of global attractor. Although in our study, we have to use classical methods of functional analysis applied in the theory of Partial Differential Equations.

\section{Results}

We first give some estimates which allow us to determine a first phase space.

\subsection{A Priory Estimates}

We a priory assume $\left\|u_{0}\right\|_{L^{\infty}(\Omega)}<1$ and $\|u\|_{L^{\infty}((0, T) \times \Omega)}<1$.

Multiplying (1) by $2 \partial_{t} u$ and (2) by $2 \partial_{t} \alpha$, integrating over $\Omega$ and adding the two resulting equations, we have

$$
\frac{d}{d t} E_{1}+2\left\|\partial_{t} u\right\|^{2}+2\left\|\partial_{t} \alpha\right\|_{H^{1}}^{2}=0
$$

where

$$
E_{1}=\epsilon\left\|\partial_{t} u\right\|^{2}+\|u\|_{H^{1}}^{2}+\left\|\partial_{t} \alpha\right\|^{2}+\|\alpha\|_{H^{1}}^{2}+2(F(u), 1),
$$

which implies, by integrating between 0 and $t$

$$
\epsilon\left\|\partial_{t} u(t)\right\|^{2}+\|u(t)\|_{H^{1}}^{2}+\left\|\partial_{t} \alpha\right\|^{2}+\|\alpha\|_{H^{1}}^{2}+\int_{0}^{t}\left\|\partial_{t} u(s)\right\|^{2} d s+\int_{0}^{t}\left\|\partial_{t} \alpha(s)\right\|_{H^{1}}^{2} d s \leq 4 c_{0}|\Omega|+K,
$$

where $K$ is a positive constant.

According to estimate $(7), u, \alpha \in L^{\infty}\left(\mathbb{R}_{+} ; H_{0}^{1}(\Omega)\right), \partial_{t} u \in L^{\infty}\left(\mathbb{R}_{+} ; L^{2}(\Omega)\right) \cap L^{2}\left(0, T ; L^{2}(\Omega)\right)$ and $\partial_{t} \alpha \in L^{\infty}\left(\mathbb{R}_{+} ; L^{2}(\Omega)\right) \cap$ $L^{2}\left(0, T ; H_{0}^{1}(\Omega)\right), \forall T>0$.

We multiply (1) by $-2 \Delta \partial_{t} u$ and (2) by $-2 \Delta \partial_{t} \alpha$, integrate over $\Omega$ and add two resulting equations. We have

$$
\begin{aligned}
\frac{d}{d t}\left(\epsilon\left\|\partial_{t} u\right\|_{H^{1}}^{2}+\|u\|_{H^{2}}^{2}+\left\|\partial_{t} \alpha\right\|_{H^{1}}^{2}+\|\alpha\|_{H^{2}}^{2}\right)+\left\|\partial_{t} u\right\|_{H^{1}}^{2}+2\left\|\partial_{t} \alpha\right\|_{H^{2}}^{2} & \leq C\|u\|_{H^{1}}^{2} \\
& \leq C\|u\|_{H^{2}}^{2} .
\end{aligned}
$$


Estimate (8) implies $u, \alpha \in L^{\infty}\left(0, T ; H^{2}(\Omega) \cap H_{0}^{1}(\Omega)\right), \partial_{t} u \in L^{\infty}\left(0, T ; H_{0}^{1}(\Omega)\right) \cap L^{2}\left(0, T ; H_{0}^{1}(\Omega)\right)$ and $\partial_{t} \alpha \in$ $L^{\infty}\left(0, T ; H_{0}^{1}(\Omega)\right) \cap L^{2}\left(0, T ; H^{2}(\Omega) \cap H_{0}^{1}(\Omega)\right), \forall T>0$. Moreover, $u$ is continuous almost for all $t \in[0, T]$.

Multiplying (1) by $2 \partial_{t}^{2} u$ and integrate over $\Omega$, we have

$$
\frac{d}{d t}\left\|\partial_{t} u\right\|^{2}+\epsilon\left\|\partial_{t}^{2} u\right\|^{2} \leq C_{1}\left\|\partial_{t} \alpha\right\|^{2}+C_{2}\|u\|_{H^{2}}^{2}+C_{3}\|f(u)\|^{2},
$$

Which implies $\partial_{t} u \in L^{2}\left(0, T ; L^{2}(\Omega)\right)$.

We multiply (2) by $-2 \Delta \partial_{t}^{2} \alpha$ and integrate over $\Omega$. We have

$$
\frac{d}{d t}\left(\left\|\partial_{t} \alpha\right\|_{H^{2}}^{2}+2\left(\Delta \alpha, \Delta \partial_{t} \alpha\right)\right)+\left\|\partial_{t}^{2} \alpha\right\|_{H^{1}}^{2} \leq\left\|\partial_{t} u\right\|_{H^{1}}^{2}+2\left\|\partial_{t} \alpha\right\|_{H^{2}}^{2} .
$$

Adding (9) and $\gamma_{1}(11)$ where $\gamma_{1}>0$, we obtain

$$
\frac{d}{d t} E_{2}+\left\|\partial_{t} u\right\|_{H^{1}}^{2}+2\left(1-\gamma_{1}\right)\left\|\partial_{t} \alpha\right\|_{H^{2}}^{2}+\gamma_{1}\left\|\partial_{t}^{2} \alpha\right\|_{H^{1}}^{2} \quad \leq \quad \gamma_{1}\left\|\partial_{t} u\right\|_{H^{1}}^{2}+C\|u\|_{H^{1}}^{2},
$$

where

$$
E_{2}=\epsilon\left\|\partial_{t} u\right\|_{H^{1}}^{2}+\|u\|_{H^{2}}^{2}+\left\|\partial_{t} \alpha\right\|_{H^{1}}^{2}+\|\alpha\|_{H^{2}}^{2}+\gamma_{1}\left(\left\|\partial_{t} \alpha\right\|_{H^{2}}^{2}+2\left(\Delta \alpha, \Delta \partial_{t} \alpha\right)\right) .
$$

We know that

$$
\|\alpha\|_{H^{2}}^{2}+\gamma_{1}\left(\left\|\partial_{t} \alpha\right\|_{H^{2}}^{2}+2\left(\Delta \alpha, \Delta \partial_{t} \alpha\right)\right) \geq\left(1-2 \gamma_{1}\right)\|\alpha\|_{H^{2}}^{2}+\frac{\gamma_{1}}{2}\left\|\partial_{t} \alpha\right\|_{H^{2}}^{2} .
$$

Choosing $\gamma_{1}$ such that $1-2 \gamma_{1}>0$, we have $E_{2} \geq 0$ and

$$
\left\|\partial_{t} \alpha\right\|_{H^{1}}^{2}+\left(1-2 \gamma_{1}\right)\|\alpha\|_{H^{2}}^{2}+\frac{\gamma_{1}}{2}\left\|\partial_{t} \alpha\right\|_{H^{2}}^{2} \leq E_{2} .
$$

According to (13), Estimate (12) implies $\partial_{t} \alpha \in L^{\infty}\left(0, T ; H^{2}(\Omega) \cap H_{0}^{1}(\Omega)\right)$ and $\partial_{t}^{2} \alpha \in L^{2}\left(0, T ; H_{0}^{1}(\Omega)\right)$. Since $H^{2}(\Omega) \subset L^{\infty}(\Omega)$ with continuous injection, then $\partial_{t} \alpha \in L^{\infty}([0, T] \times \Omega)$, there exists $c_{2}>0$ such that $\left\|\partial_{t} \alpha(t)\right\|_{L^{\infty}(\Omega)} \leq$ $c_{2} \quad \forall t \in[0, T]$, where $c_{2}$ depends of $T$ and initial conditions.

In our study there are two main results; we prove the existence and uniqueness of solution and the existence of global attractor.

\subsection{Existence and Uniqueness of Solution}

Theorem 1. (Existence) We assume $\left(u_{0}, u_{1}, \alpha_{0}, \alpha_{1}\right) \in\left(H^{2}(\Omega) \cap H_{0}^{1}(\Omega)\right) \times H_{0}^{1}(\Omega) \times\left(H^{2}(\Omega) \cap H_{0}^{1}(\Omega)\right) \times\left(H^{2}(\Omega) \cap\right.$ $\left.H_{0}^{1}(\Omega)\right)$ such that $\left\|u_{0}\right\|_{L^{\infty}(\Omega)}<1$. Then, the system $(1)-(2)$ possesses at least one solution $(u, \alpha)$ such that $u, \alpha \in L^{\infty}\left(0, T ; H^{2}(\Omega) \cap H_{0}^{1}(\Omega)\right), \quad \partial_{t} u \in L^{\infty}\left(0, T ; H_{0}^{1}(\Omega)\right) \cap L^{2}\left(0, T ; H_{0}^{1}(\Omega)\right), \quad \partial_{t} \alpha \in L^{\infty}\left(0, T ; H^{2}(\Omega) \cap H_{0}^{1}(\Omega)\right) \cap$ $L^{2}\left(0, T ; H^{2}(\Omega) \cap H_{0}^{1}(\Omega)\right), \quad \partial_{t}^{2} u \in L^{2}\left(0, T ; L^{2}(\Omega)\right)$ and $\partial_{t}^{2} \alpha \in L^{2}\left(0, T ; H_{0}^{1}(\Omega)\right), \forall T>0$. Moreover, there exists $\delta=\delta\left(T, u_{0}\right) \in(0,1)$ such that $\|u(t)\|_{L^{\infty}((0, T) \times \Omega)} \leq \delta, \forall t \in[0, T], \forall T>0$.

Proof. In order to prove this Theorem, we first show that all solution $(u, \alpha)$ of system (1) - (2) is such that $u$ is separated from the singular points of $f$, i.e., there exists $\delta \in(0,1)$ depending of $T$ such that $\|u\|_{L^{\infty}((0, T) \times \Omega)}<\delta$. In the second time, we study the auxiliary problem of the system (1) - (2). Finally, we show that the solution of auxiliary problem is also the solution of system (1) - (2).

From previous section, we have $\partial_{t} \alpha \in L^{\infty}([0, T] \times \Omega)$, then there exists $\delta \in(0,1)$ such that

$$
\left\|u_{0}\right\|_{L^{\infty}(\Omega)} \leq \delta, \quad f^{\prime}(\delta)>0 \text { and }\left\|\partial_{t} \alpha\right\|_{L^{\infty}([0, T] \times \Omega)}<f(\delta) \quad \forall t \in[0, T] .
$$

We set $U=u-\delta$ and $U^{+}=\max (U, 0)$, then $U$ satisfies the following equation

$$
\epsilon \partial_{t}^{2} U+\partial_{t} U-\Delta U+f(u)-f(\delta)=\partial_{t} \alpha-f(\delta) .
$$

Multiplying (14) par $2 \gamma_{2} U^{+}+2 \gamma_{3} \partial_{t} U^{+}$and integrating over $\Omega$, we have

$$
\frac{d}{d t} E_{3}+2 \gamma_{2}\left\|U^{+}\right\|_{H^{1}}^{2}+2 \gamma_{3}\left\|\partial_{t} U^{+}\right\|^{2} \leq 2\left(\partial_{t} \alpha-f(\delta), \gamma_{2} U^{+}+\gamma_{3} \partial_{t} U^{+}\right)+C_{1}\left(\left\|U^{+}\right\|^{2}+\left\|\partial_{t} U^{+}\right\|^{2}\right) .
$$


where

$$
E_{3}=\gamma_{2}\left(2 \epsilon\left(\partial_{t} U^{+}, U^{+}\right)+\left\|U^{+}\right\|^{2}\right)+\gamma_{3}\left(\epsilon\left\|\partial_{t} U^{+}\right\|^{2}+\left\|U^{+}\right\|_{H^{1}}^{2}\right)
$$

We have also

$$
\begin{aligned}
\gamma_{2}\left(2 \epsilon\left(\partial_{t} U^{+}, U^{+}\right)+\left\|U^{+}\right\|^{2}\right)+\gamma_{3} \epsilon\left\|\partial_{t} U^{+}\right\|^{2} & \geq \gamma_{3} \epsilon\left\|\partial_{t} U^{+}\right\|^{2}+\gamma_{2}\left(\left\|U^{+}\right\|^{2}-2\left(\epsilon^{2}\left\|\partial_{t} U^{+}\right\|^{2}+\frac{1}{4}\left\|U^{+}\right\|^{2}\right)\right) \\
& \geq \epsilon\left(\gamma_{3}-2 \epsilon \gamma_{2}\right)\left\|\partial_{t} U^{+}\right\|^{2}+\frac{\gamma_{2}}{2}\left\|U^{+}\right\|^{2} .
\end{aligned}
$$

Choosing $\gamma_{2}$ and $\gamma_{3}$ such that

$$
\begin{array}{r}
\gamma_{3}-2 \epsilon \gamma_{2}>0, \\
\gamma_{2} U^{+}(t)+\gamma_{3} \partial_{t} U^{+}(t) \geq 0 \quad \forall t \in[0, T],
\end{array}
$$

there exists $C>0$ such that

$$
C^{-1}\left(\left\|U^{+}(t)\right\|^{2}+\left\|\partial_{t} U^{+}(t)\right\|^{2}+\left\|U^{+}(t)\right\|_{H^{1}}^{2}\right) \leq E_{3}(t) \leq C\left(\left\|U^{+}(t)\right\|^{2}+\left\|\partial_{t} U^{+}(t)\right\|^{2}+\left\|U^{+}(t)\right\|_{H^{1}}^{2}\right) .
$$

Thanks to estimates (17) - (18), there exists $k>0$ such that estimate (15) implies

$$
\frac{d}{d t} E_{3}(t) \leq k E_{3}(t),
$$

which gives, using Gronwall's lemma

$$
E_{3}(t) \leq E_{3}(0) e^{k T} \quad \forall t \in[0, T]
$$

applying estimate (18) to the above estimate, we have $\forall t \in[0, T]$

$$
\left\|U^{+}(t)\right\|^{2}+\left\|\partial_{t} U^{+}(t)\right\|^{2}+\left\|U^{+}(t)\right\|_{H^{1}}^{2} \leq K\left(\left\|U^{+}(0)\right\|^{2}+\left\|\partial_{t} U^{+}(0)\right\|^{2}+\left\|U^{+}(0)\right\|_{H^{1}}^{2}\right) e^{k T}
$$

According to the estimate (18), we have $u(0)<\delta$ for almost all $x \in \Omega$, that implies $U^{+}(0)=0$. Moreover, since $u$ is continuous for almost all $t \in[0, T]$ and $u(0)<\delta$, there exists $t_{0} \in[0, T]$ such that $u(t)<\delta \quad \forall t \in\left[0, t_{0}[\right.$, this implies $U^{+}(t)=0 \quad \forall t \in\left[0, t_{0}\left[\right.\right.$ and $\partial_{t} U^{+}(0)=0$. Indeed, estimate (19) implies $U^{+}(t)=0 \quad \forall t \in[0, T]$, then $u(t) \leq \delta \quad \forall t \in[0, T]$.

In order to show $-\delta \leq u(t) \forall t \in[0, T]$, we set $V=u+\delta$ and $V^{-}=\min (V, 0)$, then $V$ satisfies the following equation

$$
\epsilon \partial_{t}^{2} V+\partial_{t} V-\Delta V+f(u)+f(\delta)=\partial_{t} \alpha+f(\delta) .
$$

Multiplying (20) by $\gamma_{4} V^{-}+\gamma_{5} \partial_{t} V^{-}$where $\gamma_{4}$ and $\gamma_{5}>0$ and integrating over $\Omega$, we have

$$
\begin{aligned}
\frac{d}{d t} E_{4}+2 \gamma_{5}\left\|\partial_{t} V^{-}\right\|^{2}+2 \gamma_{4}\left\|V^{-}\right\|_{H^{1}}^{2}+2 \gamma_{4}\left(f(u)+f(\delta), \partial_{t} V^{-}\right) & =2\left(\partial_{t} \alpha+f(\delta), \gamma_{4} V^{-}+\gamma_{5} \partial_{t} V^{-}\right) \\
& +2 \gamma_{4} \epsilon\left\|\partial_{t} V^{-}\right\|^{2},
\end{aligned}
$$

where

$$
E_{4}=\gamma_{4}\left(2 \epsilon\left(\partial_{t} V^{-}, V^{-}\right)+\left\|V^{-}\right\|^{2}\right)+\gamma_{5}\left(\epsilon\left\|\partial_{t} V^{-}\right\|^{2}+\left\|V^{-}\right\|_{H^{1}}^{2}\right)
$$

We have

$$
\begin{aligned}
\gamma_{5} \epsilon\left\|\partial_{t} V^{-}\right\|^{2}+\gamma_{4}\left(2 \epsilon\left(\partial_{t} V^{-}, V^{-}\right)+\left\|V^{-}\right\|^{2}\right) & \geq \gamma_{5} \epsilon\left\|\partial_{t} V^{-}\right\|^{2}+\gamma_{4}\left(-2\left(\epsilon^{2}\left\|\partial_{t} V^{-}\right\|^{2}+\frac{1}{4}\left\|V^{-}\right\|^{2}\right)+\left\|V^{-}\right\|^{2}\right) \\
& \geq \epsilon\left(\gamma_{5}-2 \epsilon \gamma_{4}\right)\left\|\partial_{t} V^{-}\right\|^{2}+\frac{1}{2}\left\|V^{-}\right\|^{2}
\end{aligned}
$$

Choosing $\gamma_{4}$ and $\gamma_{5}$ such that $\gamma_{5}-2 \epsilon \gamma_{4}>0$ and $\gamma_{4} V^{-}+\gamma_{5} \partial_{t} V^{-} \leq 0$ for almost $t \in[0, T]$, there exists $k>0$ such that (21) implies the following estimate

$$
\frac{d}{d t} E_{4} \leq k E_{4}
$$

Moreover, there exists $C>0$ such that

$$
C^{-1}\left(\epsilon\left\|\partial_{t} V^{-}(t)\right\|^{2}+\left\|V^{-}(t)\right\|^{2}+\left\|V^{-}(t)\right\|_{H^{1}}^{2}\right) \leq E_{4}(t) \leq C\left(\epsilon\left\|\partial_{t} V^{-}(t)\right\|^{2}+\left\|V^{-}(t)\right\|^{2}+\left\|V^{-}(t)\right\|_{H^{1}}^{2}\right) .
$$


Applying Gronwall's lemma to (22), we have, owing to estimate(23),

$$
\epsilon\left\|\partial_{t} V^{-}(t)\right\|^{2}+\left\|V^{-}(t)\right\|^{2}+\left\|V^{-}(t)\right\|_{H^{1}}^{2} \leq K\left(\epsilon\left\|\partial_{t} V^{-}(0)\right\|^{2}+\left\|V^{-}(0)\right\|^{2}+\left\|V^{-}(0)\right\|_{H^{1}}^{2}\right) e^{k T} \forall t \in[0, T] .
$$

Thanks to assumptions of Theorem, we have $-\delta<u(0)$ for almost all $x \in \Omega$, which implies $V^{-}(0)=0$. Moreover, since $u$ is continuous for almost all $t \in[0, T]$ and $-\delta<u(0)$, there exists $t_{1}>0$ such that $-\delta<u(t)$ for almost all $t \in\left[0, t_{1}\left[\right.\right.$, then $V^{-}(t)=0$ for almost all $t \in\left[0, t_{1}\left[\right.\right.$ and $\partial_{t} V^{-}(0)=0$. Indeed, estimate(24) implies $V^{-}(t)=0$ for almost all $t \in[0, T]$, then $-\delta \leq u(t)$ for almost all $t \in[0, T]$.

It then proven that there exists $\delta \in(0,1)$ such that $\|u(t)\|_{L^{\infty}((0, T) \times \Omega)} \leq \delta$.

We now have to prove the existence of a solution $\left(u^{\delta}, \alpha\right)$ of the following auxiliary system

$$
\begin{aligned}
\epsilon \frac{\partial^{2} u^{\delta}}{\partial t^{2}}+\frac{\partial u^{\delta}}{\partial t}-\Delta u^{\delta}+f_{\delta}\left(u^{\delta}\right) & =\frac{\partial \alpha}{\partial t} \\
\frac{\partial^{2} \alpha}{\partial t^{2}}-\frac{\partial \Delta \alpha}{\partial t}-\Delta \alpha & =-\frac{\partial u^{\delta}}{\partial t}
\end{aligned}
$$

obtained by replacing logarithmic function $f$ by the $C^{1}(\mathbb{R})$-regular function $f_{\delta}$ in $(1), f_{\delta}$ being defined by

$$
f_{\delta}(s)=\left\{\begin{array}{cc}
f(-\delta)+f^{\prime}(-\delta)(s+\delta) & , s<-\delta \\
f(s) & ,|s| \leq \delta \\
f(\delta)+f^{\prime}(\delta)(s-\delta) & , s>\delta
\end{array}\right.
$$

where $\delta>0$ very near of 1 and such that $[-\delta, \delta] \subset(-1,1)$, with homogenous Dirichlet conditions and initial conditions in (3) - (4).

The existence of a solution of system (25) - (26) is based on estimates (27) - (30) below and a standard Galerkin scheme (see (Moukoko 2015)).

Multiplying (25) by $2 \partial_{t} u^{\delta}$ and (26) by $2 \partial_{t} \alpha$ integrating over $\Omega$ adding the two resulting equations, we have

$$
\frac{d}{d t}\left(\epsilon\left\|\partial_{t} u^{\delta}\right\|^{2}+\left\|u^{\delta}\right\|^{2}+\left\|\partial_{t} \alpha\right\|^{2}+\|\alpha\|^{2}+2\left(F_{\delta}\left(u^{\delta}\right), 1\right)\right)+2\left\|\partial_{t} u^{\delta}\right\|^{2}+2\left\|\partial_{t} \alpha\right\|_{H^{1}}^{2}=0 .
$$

The above estimate implies $u^{\delta}, \alpha \in L^{\infty}\left(\mathbb{R} ; H_{0}^{1}(\Omega)\right), \partial_{t} u^{\delta} \in L^{\infty}\left(\mathbb{R} ; L^{2}(\Omega)\right) \cap L^{2}\left(\mathbb{R} ; L^{2}(\Omega)\right)$ and $\partial_{t} \alpha \in L^{\infty}\left(\mathbb{R} ; L^{2}(\Omega)\right) \cap$ $L^{2}\left(\mathbb{R} ; H_{0}^{1}(\Omega)\right.$.

Multiplying (25) by $-2 \Delta \partial_{t} u^{\delta}$ and (26) by $-2 \Delta \partial_{t} \alpha$, integrating over $\Omega$ and adding the two resulting equations, we obtain

$$
\frac{d}{d t} E_{5}+\left\|\partial_{t} u^{\delta}\right\|_{H^{1}}^{2}+2\left\|\partial_{t} \alpha\right\|_{H^{2}}^{2} \leq C\left\|u^{\delta}\right\|_{H^{1}}^{2}
$$

where

$$
E_{5}=\epsilon\left\|\partial_{t} u^{\delta}\right\|_{H^{1}}^{2}+\left\|u^{\delta}\right\|_{H^{2}}^{2}+\left\|\partial_{t} \alpha\right\|_{H^{1}}^{2}+\|\alpha\|_{H^{2}}^{2} .
$$

Therefore $u^{\delta}, \alpha \in L^{\infty}\left(0, T ; H^{2}(\Omega) \cap H_{0}^{1}(\Omega)\right), \partial_{t} u^{\delta} \in L^{\infty}\left(0, T ; H_{0}^{1}(\Omega)\right) \cap L^{2}\left(0, T ; H_{0}^{1}(\Omega)\right)$ and $\partial_{t} \alpha \in L^{\infty}\left(0, T ; H_{0}^{1}(\Omega)\right) \cap$ $L^{2}\left(0, T ; H^{2}(\Omega) \cap H_{0}^{1}(\Omega)\right)$.

Multiplying (25) by $2 \partial_{t}^{2} u^{\delta}$ and integrating over $\Omega$, we obtain

$$
\frac{d}{d t}\left\|\partial_{t} u^{\delta}\right\|^{2}+\epsilon\left\|\partial_{t}^{2} u^{\delta}\right\|^{2} \leq C_{1}\left\|u^{\delta}\right\|_{H^{2}}^{2}+C_{2}\left\|f_{\delta}\left(u^{\delta}\right)\right\|^{2}+C_{3}\left\|\partial_{t} \alpha\right\|^{2},
$$

therefore $\partial_{t}^{2} u^{\delta} \in L^{2}\left(0, T ; L^{2}(\Omega)\right)$.

Multiplying (26) by $-2 \Delta \partial_{t}^{2} \alpha$ and integrate over $\Omega$, we have

$$
\frac{d}{d t}\left(\left\|\partial_{t} \alpha\right\|_{H^{2}}^{2}+2\left(\Delta \alpha, \Delta \partial_{t} \alpha\right)\right)+\left\|\partial_{t}^{2} \alpha\right\|_{H^{1}}^{2} \leq\left\|\partial_{t} u^{\delta}\right\|_{H^{1}}^{2}+2\left\|\partial_{t} \alpha\right\|_{H^{2}}^{2} .
$$

Adding (29) and $\gamma_{6}(28)$ where $\gamma_{6}>0$, we have

$$
\begin{aligned}
\frac{d}{d t}\left(\epsilon\left\|\partial_{t} u^{\delta}\right\|_{H^{1}}^{2}+\left\|\Delta u^{\delta}\right\|_{H^{2}}^{2}+\left\|\partial_{t} \alpha\right\|_{H^{1}}^{2}\right. & \left.+\|\alpha\|^{2}+\gamma_{6}\left\|\partial_{t} \alpha\right\|_{H^{2}}^{2}+2 \gamma_{6}\left(\Delta \alpha, \Delta \partial_{t} \alpha\right)\right)+\gamma_{6}\left\|\partial_{t}^{2} \alpha\right\|_{H^{1}}^{2} \\
& +2\left(1-\gamma_{6}\right)\left\|\partial_{t} \alpha\right\|_{H^{2}}^{2}+\left(1-\gamma_{6}\right)\left\|\partial_{t} u^{\delta}\right\|_{H^{1}}^{2} \leq C\left\|u^{\delta}\right\|_{H^{2}}^{2} .
\end{aligned}
$$


We have

$$
\|\alpha\|_{H^{2}}^{2}+\gamma_{6}\left\|\partial_{t} \alpha\right\|_{H^{2}}^{2}+2 \gamma_{6}\left(\Delta \alpha, \Delta \partial_{t} \alpha \geq\left(1-2 \gamma_{6}\right)\|\alpha\|_{H^{2}}^{2}+\frac{\gamma_{6}}{2}\left\|\partial_{t} \alpha\right\|_{H^{2}}^{2} .\right.
$$

We choose $\gamma_{6}<\frac{1}{2}$, then (29) implies $\partial_{t} \alpha \in L^{\infty}\left(0, T ; H^{2}(\Omega) \cap H_{0}^{1}(\Omega)\right) \cap L^{2}\left(0, T ; H^{2}(\Omega) \cap H_{0}^{1}(\Omega)\right)$ et $\partial_{t}^{2} \alpha \in$ $L^{2}\left(0, T ; H_{0}^{1}(\Omega)\right)$. Therefore, the auxiliary system possesses a solution $\left(u^{\delta}, \alpha\right)$ such that $u^{\delta}, \alpha \in L^{\infty}\left(0, T ; H^{2}(\Omega) \cap\right.$ $\left.H_{0}^{1}(\Omega)\right), \partial_{t} u^{\delta} \in L^{2}\left(0, T ; H_{0}^{1}(\Omega)\right), \partial_{t} \alpha \in L^{\infty}\left(0, T ; H^{2}(\Omega) \cap H_{0}^{1}(\Omega)\right) \cap L^{2}\left(0, T ; H^{2}(\Omega) \cap H_{0}^{1}(\Omega)\right), \partial_{t}^{2} u \in L^{2}\left(0, T ; L^{2}(\Omega)\right.$ and $\partial_{t}^{2} \alpha \in L^{2}\left(0, T ; H_{0}^{1}(\Omega)\right), \forall T>0$.

Since

$$
\left\|u_{0}\right\|_{L^{\infty}(\Omega)}<\delta, \quad f_{\delta}^{\prime}(\delta)>0 \text { and }\left\|\partial_{t} \alpha\right\|_{L^{\infty}((0, T) \times \Omega)}<f_{\delta}(\delta),
$$

then $\left\|u^{\delta}\right\|_{L^{\infty}((0, T) \times \Omega)} \leq \delta$, then $f_{\delta}\left(u^{\delta}\right)=f\left(u^{\delta}\right)$ and $\left(u^{\delta}, \alpha\right)$ is solution of system (1)-(2).

Theorem 2. (uniqueness) Let the assumptions of Theorem 1 hold. Then, system (1)-(2) possesses a unique solution $(u, \alpha)$ such that $u, \alpha \in L^{\infty}\left(0, T ; H^{2}(\Omega) \cap H_{0}^{1}(\Omega)\right), \quad \partial_{t} u \in L^{2}\left(0, T ; H_{0}^{1}(\Omega)\right), \quad \partial_{t} \alpha \in L^{\infty}\left(0, T ; H^{2}(\Omega) \cap\right.$ $\left.H_{0}^{1}(\Omega)\right) \cap L^{2}\left(0, T ; H^{2}(\Omega) \cap H_{0}^{1}(\Omega)\right), \quad \partial_{t}^{2} u \in L^{2}\left(0, T ; L^{2}(\Omega), \partial_{t}^{2} \alpha \in L^{2}\left(0, T ; H_{0}^{1}(\Omega)\right), \forall T>0\right.$.

Proof. Let be $T>0$ and $\left(u^{(i)}, \alpha^{(i)}\right)_{i=1,2}$ two solutions of system (1)-(2) with initial conditions $\left(u_{0}^{(i)}, \alpha_{0}^{(i)}, u_{1}^{(i)}, \alpha_{1}^{(i)}\right)_{i=1,2}$ respectively, such that $\left\|u^{(i)}(0)\right\|_{L^{\infty}(\Omega)}<\delta^{(i)}<1$ for $i=1,2$.

We set $u=u^{(1)}-u^{(2)}$ and $\alpha=\alpha^{(1)}-\alpha^{(2)}$. Then, $(u, \alpha)$ satisfies the following system

$$
\begin{aligned}
\epsilon \partial_{t}^{2} u+\partial_{t} u-\Delta u+l(t) u & =\partial_{t} \alpha \\
\partial_{t}^{2} \alpha-\partial_{t} \Delta \alpha-\Delta \alpha & =-\partial_{t} u
\end{aligned}
$$

where $l(t)=\int_{0}^{1} f^{\prime}\left(s u_{1}(t)+(1-s) u_{2}(t)\right) d s$, with homogenous Dirichlet conditions and following initial conditions

$$
\begin{array}{ll}
u(0, x)=u_{0}=u_{0}^{(1)}-u_{0}^{(2)} & \alpha(0, x)=\alpha_{0}=\alpha_{0}^{(1)}-\alpha_{0}^{(2)} \\
\left.\partial_{t} u\right|_{t=0}=u_{1}=u_{1}^{(1)}-u_{1}^{(2)} & \left.\partial_{t} \alpha\right|_{t=0}=\alpha_{1}=\alpha_{1}^{(1)}-\alpha_{1}^{(2)},
\end{array}
$$

Since $f \in C^{2}(-1,1)$ and $u_{i}$ are separated from $\pm 1, \quad i=1,2$, we have

$$
\|l(t)\|_{L^{\infty}} \leq c, \forall t \geq 0
$$

where $c$ depends of $\delta_{i}, i=1,2$.

Multiplying (31) by $2 \partial_{t} u$ and (32) by $2 \partial_{t} \alpha$, integrating over $\Omega$ and adding two resulting equations, we have

$$
\frac{d}{d t}\left(\epsilon\left\|\partial_{t} u\right\|^{2}+\|u\|_{H^{1}}^{2}+\left\|\partial_{t} \alpha\right\|^{2}+\|\alpha\|_{H^{1}}^{2}\right)+\left\|\partial_{t} u\right\|^{2}+2\left\|\partial_{t} \alpha\right\|_{H^{1}}^{2} \leq C\|u\|_{H^{1}}^{2}
$$

where $C$ depends of $\delta_{i}$. Applying Gronwall's lemma to (33), we have

$$
\epsilon\left\|\partial_{t} u(t)\right\|^{2}+\|u(t)\|_{H^{1}}^{2}+\left\|\partial_{t} \alpha(t)\right\|^{2}+\|\alpha(t)\|_{H^{1}}^{2} \leq e^{C t}\left(\epsilon\left\|\partial_{t} u(0)\right\|^{2}+\|u(0)\|_{H^{1}}^{2}+\left\|\partial_{t} \alpha(0)\right\|^{2}+\|\alpha(0)\|_{H^{1}}^{2}\right) .
$$

We have the continuous dependence with respect to initial conditions, hence the uniqueness of solution.

In order to prove the existence of global attractor we seek the solution with more regularity.

Theorem 3. Assume $\left(u_{0}, u_{1}, \alpha_{0}, \alpha_{1}\right) \in\left(H^{3}(\Omega) \cap H_{0}^{1}(\Omega)\right) \times\left(H^{2}(\Omega) \cap H_{0}^{1}(\Omega)\right) \times\left(H^{3}(\Omega) \cap H_{0}^{1}(\Omega)\right) \times\left(H^{3}(\Omega) \cap H_{0}^{1}(\Omega)\right)$ and $\left\|u_{0}\right\|_{L^{\infty}(\Omega)}<1$. Then (1)-(2) possesses a unique solution $(u, \alpha)$ such that $u, \alpha \in L^{\infty}\left(0, T ; H^{3}(\Omega) \cap H_{0}^{1}(\Omega)\right)$, $\partial_{t} u \in$ $L^{\infty}\left(0, T ;\left(H^{2}(\Omega) \cap H_{0}^{1}(\Omega)\right)\right) \cap L^{2}\left(0, T ; H^{2}(\Omega) \cap H_{0}^{1}(\Omega)\right), \partial_{t} \alpha \in L^{\infty}\left(0, T ; H^{3}(\Omega) \cap H_{0}^{1}(\Omega)\right) \cap L^{2}\left(0, T ; H^{3}(\Omega) \cap H_{0}^{1}(\Omega)\right)$ and $\partial_{t}^{2} \alpha \in L^{2}\left(0, T ; H^{2}(\Omega) \cap H_{0}^{1}(\Omega)\right), \forall T>0$. Moreover, there exists $\delta=\delta\left(T, u_{0}\right) \in(0,1)$ such that $\|u(t)\|_{L^{\infty}((0, T) \times \Omega)}<$ $\delta, \forall t \in[0, T], \forall T>0$.

Proof. Following Theorem 1, system (1) - (2) has a unique solution $(u, \alpha)$ such that $u, \alpha \in L^{\infty}\left(0, T ; H^{2}(\Omega) \cap\right.$ $\left.H_{0}^{1}(\Omega)\right), \quad \partial_{t} u \in L^{2}\left(0, T ; H_{0}^{1}(\Omega)\right)$ and $\partial_{t}^{2} \alpha \in L^{2}\left(0, T ; L^{2}(\Omega)\right), \forall T>0$.

Multiplying (1) by $2 \Delta^{2} \partial_{t} u$ and (2) by $2 \Delta^{2} \partial_{t} \alpha$, integrating over $\Omega$ and adding the two resulting equations, we obtain

$$
\frac{d}{d t}\left(\epsilon\left\|\partial_{t} u\right\|_{H^{2}}^{2}+\|\nabla \Delta u\|^{2}+\left\|\partial_{t} \alpha\right\|_{H^{2}}^{2}+\|\nabla \Delta \alpha\|^{2}\right)+\left\|\partial_{t} u\right\|_{H^{2}}^{2}+2\left\|\nabla \Delta \partial_{t} \alpha\right\|^{2} \leq C .
$$


Therefore $u, \alpha \in L^{\infty}\left(0, T ; H^{3}(\Omega) \cap H_{0}^{1}(\Omega)\right), \quad \partial_{t} u \in L^{\infty}\left(0, T ; H^{2}(\Omega) \cap H_{0}^{1}(\Omega)\right) \cap L^{2}\left(0, T ; H^{2}(\Omega) \cap H_{0}^{1}(\Omega)\right), \quad \partial_{t} \alpha \in$ $L^{\infty}\left(0, T ; H^{2}(\Omega) \cap H_{0}^{1}(\Omega)\right) \cap L^{2}\left(0, T ; H^{3}(\Omega) \cap H_{0}^{1}(\Omega)\right)$.

Multiply (2) by $-2 \Delta^{2} \partial_{t}^{2} \alpha$ and integrate over $\Omega$. We have, owing the continuous injections $H^{2}(\Omega) \subset L^{\infty}(\Omega)$ and $H^{3}(\Omega) \subset L^{\infty}(\Omega)$,

$$
\frac{d}{d t}\left\|\nabla \Delta \partial_{t} \alpha\right\|^{2}+\left\|\partial_{t}^{2} \alpha\right\|_{H^{2}}^{2} \leq C,
$$

which implies $\partial_{t} \alpha \in L^{\infty}\left(0, T ; H^{3}(\Omega) \cap H_{0}^{1}(\Omega)\right) \cap L^{2}\left(0, T ; H^{3}(\Omega) \cap H_{0}^{1}(\Omega)\right)$ and $\partial_{t}^{2} \alpha \in L^{2}\left(0, T ; H^{2}(\Omega) \cap H_{0}^{1}(\Omega)\right)$. This finishes the proof of the Theorem.

\subsection{Existence of Global Attractor}

The phase-field spaces are $\Phi_{\kappa}=\left\{(u, v, \alpha, \beta) \in\left(H^{\kappa}(\Omega) \cap H_{0}^{1}(\Omega)\right) \times\left(H^{\kappa-1}(\Omega) \cap H_{0}^{1}(\Omega)\right) \times\left(H^{\kappa}(\Omega) \cap H_{0}^{1}(\Omega)\right) \times\left(H^{\kappa}(\Omega) \cap\right.\right.$ $\left.\left.H_{0}^{1}(\Omega)\right):\|u\|_{L^{\infty}((0, T) \times \Omega)}<1\right\}$ for $\kappa=2,3$, with energy norm

$$
\left\|\left(u, \partial_{t} u, \alpha, \partial_{t} \alpha\right)\right\|_{\Phi_{\kappa}}^{2}=\left\|\zeta_{u}\right\|_{\mathcal{E}^{k}(\epsilon)}^{2}+\|\alpha\|_{H^{k}}^{2}+\left\|\partial_{t} \alpha\right\|_{H^{k}}^{2} .
$$

Thanks to theorems 1 and 3, we define the semigroup of operators $S_{t}(\epsilon)$ resolving the system (1) - (2) by

$$
S_{t}(\epsilon): \Phi_{\kappa} \longrightarrow \Phi_{\kappa} \quad\left(u_{0}, u_{1}, \alpha_{0}, \alpha_{1}\right) \longmapsto\left(u(t), \partial_{t} u(t), \alpha(t), \partial_{t} \alpha(t)\right),
$$

where $\left(u(t), \partial_{t} u(t), \alpha(t), \partial_{t} \alpha(t)\right)$ is such that $(u, \alpha)$ is uniqueness solution of phase-field system (1) - (2) for initial conditions $\left(u_{0}, u_{1}, \alpha_{0}, \alpha_{1}\right) \in \Phi_{\kappa}$.

The following lemma gives the uniform estimates of $\|u(t)\|_{H^{2}},\|\alpha(t)\|_{H^{2}}$ and $\left\|\partial_{t} \alpha(t)\right\|_{H^{2}}$ independent of $\epsilon$, which allow to prove dissipativity of semigroup $S_{t}(\epsilon)$ in $\Phi_{2}$.

Lemma 1. Let the assumptions of Theorem 1 hold, $\epsilon<1$ and $(u, \alpha)$ the solution of system (1) - (2) such that $\left(u(0), \partial_{t} u(0), \alpha(0), \partial_{t} \alpha(0)\right) \in \Phi_{2}$. Then, the solution $(u, \alpha)$ verifies the following estimate

$$
\begin{aligned}
& \mathbb{D}[u(t)]+\|u(t)\|_{H^{2}}^{2}+\epsilon\left\|\partial_{t} u(t)\right\|_{H^{1}}^{2}+\|\alpha(t)\|_{H^{2}}^{2}+\left\|\partial_{t} \alpha(t)\right\|_{H^{2}}^{2} \\
& +\int_{0}^{t} e^{-\beta(t-s)}\left(\left\|\partial_{t} u(s)\right\|_{H^{1}}^{2}+\left\|\partial_{t}^{2} \alpha(s)\right\|_{H^{1}}^{2}\right) d s \leq Q\left(\mathbb{D}[u(0)],\left\|\zeta_{u}(0)\right\|_{\varepsilon^{2}(\epsilon)},\|\alpha(0)\|_{H^{2}},\left\|\partial_{t} \alpha(0)\right\|_{H^{2}}\right) e^{-\beta t}+C,
\end{aligned}
$$

where the positive constants $C, \beta$ and the monotonic function $Q$ are independent of $\epsilon$.

Proof. Multiply (1) by $-2 \Delta u$ and (2) by $-2 \Delta \alpha$ and integrate over $\Omega$. We obtain, thanks to continuous injection $H^{2}(\Omega) \subset L^{\infty}(\Omega)$

$$
\begin{aligned}
\frac{d}{d t}\left(2 \epsilon\left(\nabla \partial_{t} u, \nabla u\right)+\|u\|_{H^{2}}^{2}\right)+\|u\|_{H^{2}}^{2} & \leq 2\left\|\partial_{t} u\right\|_{H^{1}}^{2}+C, \\
\frac{d}{d t}\|\alpha\|_{H^{2}}^{2}+\|\alpha\|_{H^{2}}^{2} & \leq C_{2}\left\|\partial_{t} u\right\|_{H^{1}}^{2}+C_{3}\left\|\partial_{t}^{2} \alpha\right\|_{H^{1}}^{2} .
\end{aligned}
$$

Adding $\gamma_{7}(9), \gamma_{8}(11), \gamma_{9}(36)$ et $\gamma_{10}(37)$ where $\gamma_{7}, \gamma_{8}, \gamma_{9}$ and $\gamma_{10}>0$ are such that

$$
\begin{gathered}
\gamma_{7}-\gamma_{8}-2 \gamma_{9}-C_{2} \gamma_{10}>0, \\
2 \gamma_{7}-2 \gamma_{8}>0 \\
\gamma_{8}-C_{3} \gamma_{10}>0 \\
\gamma_{9}-C \gamma_{7}>0
\end{gathered}
$$

we have

$$
\frac{d}{d t} E_{6}+C_{1}\left\|\partial_{t} u\right\|_{H^{1}}^{2}+C_{2}\|u\|_{H^{2}}^{2}+2 \gamma_{9}\|\alpha\|_{H^{2}}^{2}+C_{3}\left\|\partial_{t} \alpha\right\|_{H^{2}}^{2}+C_{4}\left\|\partial_{t}^{2} \alpha\right\|_{H^{1}}^{2} \leq C,
$$

where

$$
\begin{aligned}
E_{6}= & \gamma_{7}\left(\epsilon\left\|\partial_{t} u\right\|_{H^{1}}^{2}+\|u\|_{H^{2}}^{2}+\left\|\partial_{t} \alpha\right\|_{H^{1}}^{2}+\|\alpha\|_{H^{2}}^{2}\right)+\gamma_{8}\left(\left\|\partial_{t} \alpha\right\|_{H^{2}}^{2}+2\left(\Delta \alpha, \Delta \partial_{t} \alpha\right)\right) \\
& +\gamma_{9}\left(2 \epsilon\left(\nabla \partial_{t} u, \nabla u\right)+\|u\|_{H^{2}}^{2}\right)+\gamma_{10}\|\alpha\|_{H^{2}}^{2} .
\end{aligned}
$$


There exists $\beta>0$ such that

$$
\beta E_{6} \leq \frac{C_{1}}{2}\left\|\partial_{t} u\right\|_{H^{1}}^{2}+C_{2}\|u\|_{H^{2}}^{2}+2 \gamma_{9}\|\alpha\|_{H^{2}}^{2}+C_{3}\left\|\partial_{t} \alpha\right\|_{H^{2}}^{2} .
$$

Thanks to above estimate, estimate (38) can be written on form

$$
\frac{d}{d t} E_{6}+\beta E_{6}+\frac{C_{1}}{2}\left\|\partial_{t} u\right\|_{H^{1}}^{2}+C_{4}\left\|\partial_{t}^{2} \alpha\right\|_{H^{1}}^{2} \leq C,
$$

which implies, thanks to Gronwall's lemma,

$$
E_{6}(t)+\int_{0}^{t} e^{\beta(t-s)}\left(\left\|\partial_{t} u\right\|_{H^{1}}^{2}+\left\|\partial_{t}^{2} \alpha(s)\right\|_{H^{1}}^{2}\right) d s \leq E_{6}(0) e^{-\beta t}+C .
$$

Moreover, for very small values of $\gamma_{8}$ and $\gamma_{9}$, there exists $C>0$ independent of $\epsilon$ such that

$$
\left.C^{-1}\left(\epsilon\left\|\partial_{t} u(t)\right\|_{H^{1}}^{2}+\|u(t)\|_{H^{2}}^{2}+\|\alpha(t)\|_{H^{2}}^{2}+\| \partial_{t} \alpha(t)\right) \|_{H^{2}}^{2}\right) \leq E_{6}(t) \leq C\left\|\left(u(t), \partial_{t} u(t), \alpha(t), \partial_{t} \alpha(t)\right)\right\|_{\Phi_{2}}^{2} .
$$

Thanks to above estimate, (39) implies

$$
\begin{aligned}
& \left.\mathbb{D}[u(t)]+\epsilon\left\|\partial_{t} u(t)\right\|_{H^{1}}^{2}+\|u(t)\|_{H^{2}}^{2}+\|\alpha(t)\|_{H^{2}}^{2}+\| \partial_{t} \alpha(t)\right) \|_{H^{2}}^{2} \\
& +\int_{0}^{t} e^{\beta(t-s)}\left(\left\|\partial_{t} u(s)\right\|_{H^{1}}^{2}+\left\|\partial_{t}^{2} \alpha(s)\right\|_{H^{1}}^{2}\right) d s \leq Q\left(\mathbb{D}[u(0)],\left\|\zeta_{u}(0)\right\|_{\varepsilon^{2}(\epsilon)},\|\alpha(0)\|_{H^{2}},\left\|\partial_{t} \alpha(0)\right\|_{H^{2}}\right) e^{-\beta t}+C,
\end{aligned}
$$

this achieves the proof.

Theorem 4. Let the assumptions of Theorem 1 hold, $\epsilon<1$ and $(u, \alpha)$ the solution of system (1) - (2) such that $\left(u(0), \partial_{t} u(0), \alpha(0), \partial_{t} \alpha(0)\right) \in \Phi_{2}$. Then, the following estimate is valid

$$
\begin{aligned}
& \left\|\left(\zeta_{u}(t), \alpha(t), \partial_{t} \alpha(t)\right)\right\|_{\Phi_{2}}^{2}+\int_{0}^{t} e^{-\beta(t-s)}\left(\left\|\partial_{t} u(s)\right\|^{2}+\left\|\partial_{t} \alpha(s)\right\|^{2}+\left\|\partial_{t} \alpha(s)\right\|_{H^{1}}^{2}\right) d s \\
& \leq Q\left(\mathbb{D}[u(0)],\left\|\zeta_{u}(0)\right\|_{\varepsilon^{2}(\epsilon)},\|\alpha(0)\|_{H^{2}},\left\|\partial_{t} \alpha(0)\right\|_{H^{2}}\right) e^{-\beta t}+C,
\end{aligned}
$$

where positive constants $C, \beta$ and monotonic function $Q$ are independent of $\epsilon$.

Proof. We first determine standard energy of the initial and boundary value problem for singularly perturbed damped hyperbolic equation (1). This equation can be written in the following form

$$
\epsilon \partial_{t}^{2} u+\partial_{t} u-\Delta u=-f(u(t))+\partial_{t} \alpha(t)=h_{u, \alpha}(t),\left.\quad u(t)\right|_{\partial \Omega}=\left.h(t)\right|_{\partial \Omega}=0 .
$$

Applying corollary 5.2 of appendix of (Grasselli, Miranville, Pata \& Zelik 2007) to equation (41) where $\kappa=2$, we have

$$
\begin{aligned}
\left\|\zeta_{u}(t)\right\|_{\mathcal{E}^{2}(\epsilon)}^{2}+\int_{0}^{t} e^{-\beta(t-s)}\left\|\partial_{t} u(s)\right\|_{H^{2}}^{2} d s & \leq C e^{-\beta t}\left(\left\|\zeta_{u}(0)\right\|_{\mathcal{E}^{2}(\epsilon)}^{2}+\left\|h_{u, \alpha}(0)\right\|_{H^{1}}^{2}\right) \\
& +C \int_{0}^{t} e^{-\beta(t-s)}\left(\left\|h_{u, \alpha}(s)\right\|_{H^{2}}^{2}+\left\|\partial_{t} h_{u, \alpha}(s)\right\|^{2}\right) d s,
\end{aligned}
$$

where positive constants $C$ and $\beta$ are independent of $\epsilon$.

To determine estimate of the last term of second member of (42), we begin by finding an estimate of $\left\|h_{u, \alpha}(s)\right\|_{H^{2}}^{2}$ $+\left\|\partial_{t} h_{u, \alpha}(s)\right\|^{2}$. We have, indeed,

$$
\begin{aligned}
\left\|h_{u, \alpha}(s)\right\|_{H^{2}}^{2}+\left\|\partial_{t} h_{u, \alpha}(s)\right\|^{2} & \leq 2\|f(u(s))\|_{H^{2}}^{2}+2\left\|\partial_{t} \alpha(s)\right\|_{H^{2}}^{2}+2\left\|f^{\prime}(u(s)) \partial_{t} u(s)\right\|^{2} \\
& +2\left\|\partial_{t}^{2} \alpha(s)\right\|^{2} .
\end{aligned}
$$

We now determine estimates of $\|f(u)\|_{H^{2}}$ and $\left\|f^{\prime}(u) \partial_{t} u\right\|$. We have

$$
\|f(u)\|_{H^{2}}=\left\|f^{\prime \prime}(u)(\nabla u)^{2}+f^{\prime}(u) \Delta u\right\| \leq C\left(\|u\|_{H^{2}}^{2}+\|u\|_{H^{2}}\right) \leq C,
$$

where $C$ is independent of $\epsilon$. We have also

$$
\left\|f^{\prime}(u) \partial_{t} u\right\| \leq\left\|f^{\prime}(u)\right\|_{L^{\infty}}\left\|\partial_{t} u\right\| \leq C\left\|\partial_{t} u\right\|,
$$


where $C$ is independent of $\epsilon$. We have, allow for estimate (7),

$$
\left\|h_{u, \alpha}(s)\right\|_{H^{2}}^{2}+\left\|\partial_{t} h_{u, \alpha}(s)\right\|^{2} \leq C\left(1+\left\|\partial_{t} u(s)\right\|_{H^{1}}^{2}+\left\|\partial_{t}^{2} \alpha(s)\right\|_{H^{1}}^{2}\right) .
$$

Inserting (44) into (42), we obtain, thanks to estimate (35),

$$
\begin{aligned}
& \left\|\zeta_{u}(t)\right\|_{\varepsilon^{2}(\epsilon)}^{2}+\int_{0}^{t} e^{-\beta(t-s)}\left\|\partial_{t} u(s)\right\|^{2} d s \leq \\
& C e^{-\beta t}\left(\left\|\zeta_{u}(0)\right\|_{\mathcal{\varepsilon}^{2}(\epsilon)}^{2}+\left\|h_{u, \alpha}(0)\right\|_{H^{1}}^{2}\right)+C \int_{0}^{t} e^{-\beta(t-s)}\left(1+\left\|\partial_{t} u\right\|_{H^{1}}^{2}+\left\|\partial_{t}^{2} \alpha(s)\right\|_{H^{1}}^{2}\right) d s \\
& \leq C e^{-\beta t}\left(\left\|\zeta_{u}(0)\right\|_{\varepsilon^{2}(\epsilon)}^{2}+\left\|h_{u, \alpha}(0)\right\|_{H^{1}}^{2}\right)+Q\left(\mathbb{D}[u](0),\left\|\left(\zeta_{u}(0), \alpha(0), \partial_{t} \alpha(0)\right)\right\|_{\Phi_{1}}\right) e^{-\beta t} \\
& \leq Q\left(\mathbb{D}[u(0)],\left\|\zeta_{u}(0)\right\|_{\varepsilon^{2}(\epsilon)},\|\alpha(0)\|_{H^{2}},\left\|\partial_{t} \alpha(0)\right\|_{H^{2}}\right) e^{-\beta t}+C,
\end{aligned}
$$

where positives constants $C$ and $\beta$ are independent of $\epsilon$. Combining above estimate and estimate (35), we obtain the result. This achieves the proof.

Corollary 1. The semigroup $S_{t}(\epsilon)$ associated with system (1) - (2) is dissipative in $\Phi_{2}$, i.e., it possesses a bounded absorbing set in $\Phi_{2}$.

This corollary is the straightforward consequence of above Theorem.

In the sequence, we note $\mathcal{B}_{R_{0}}^{1}(\epsilon)=\left\{(u, v, \alpha, v) \in \Phi_{2} ;\|(u, v, \alpha, \omega)\|_{\Phi_{2}} \leq R_{0}\right\}$ where $R_{0}$ is enough great, a bounded absorbing set in $\Phi_{2}$.

Theorem 5. Let the assumptions of Theorem 3 hold, $\epsilon<1$ and $(u, \alpha)$ the solution of system (1) - (2) such that $\left(u(0), \partial_{t} u(0), \alpha(0), \partial_{t} \alpha(0)\right) \in \mathcal{B}_{R_{0}}^{1}(\epsilon) \cap \Phi_{3}$. Then, following estimate is verified

$$
\begin{aligned}
& \mathbb{D}[u](t)+\left\|\left(u(t), \partial_{t} u(t), \alpha(t), \partial_{t} \alpha(t)\right)\right\|_{\Phi_{3}}^{2}+\int_{0}^{t} e^{-\beta(t-s)}\left(\left\|\partial_{t} u(s)\right\|_{H^{1}}^{2}+\epsilon\left\|\partial_{t}^{2} u(s)\right\|_{H^{1}}^{2}+\left\|\partial_{t}^{2} \alpha(s)\right\|_{H^{1}}^{2}\right) d s \\
& \leq Q\left(\mathbb{D}[u(0)],\left\|\zeta_{u}(0)\right\|_{\varepsilon^{3}(\epsilon)},\|\alpha(0)\|_{H^{3}},\left\|\partial_{t} \alpha(0)\right\|_{H^{3}}\right) e^{-\beta t}+C,
\end{aligned}
$$

where the positive constants $C, \beta$ and the monotonic function $Q$ are independent of $\epsilon$.

Proof. Multiplying (1) by $2 \Delta^{2} \partial_{t} u$ and (2) by $2 \Delta^{2} \partial_{t} \alpha$, integrating over $\Omega$ and adding the resulting equations, we have

$$
\frac{d}{d t}\left(\epsilon\left\|\partial_{t} u\right\|_{H^{2}}^{2}+\|\nabla \Delta u\|^{2}+\left\|\partial_{t} \alpha\right\|_{H^{2}}^{2}+\|\nabla \Delta \alpha\|^{2}\right)+\left\|\partial_{t} u\right\|_{H^{2}}^{2}+2\left\|\nabla \Delta \partial_{t} \alpha\right\|^{2} \leq C .
$$

Multiply (1) by $2 \Delta^{2} u$ and (2) by $2 \Delta^{2} \alpha$, and integrate over $\Omega$. We obtain

$$
\begin{aligned}
\frac{d}{d t}\left(2 \epsilon\left(\Delta \partial_{t} u, \Delta u\right)+\|u\|_{H^{2}}^{2}\right)+\|\nabla \Delta u\|^{2} & \leq C \\
\frac{d}{d t}\left(2\left(\Delta \partial_{t} \alpha, \Delta \alpha\right)+\|\alpha\|_{H^{3}}^{2}\right)+\|\nabla \Delta \alpha\|^{2} & \leq\left\|\partial_{t} u\right\|_{H^{1}}^{2}+C .
\end{aligned}
$$

Multiply (2) by $2 \Delta^{2} \partial_{t}^{2} \alpha$ and integrate over $\Omega$. We find

$$
\frac{d}{d t}\left(\left\|\partial_{t} \alpha\right\|_{H^{3}}^{2}+2\left(\nabla \Delta \alpha, \nabla \Delta \partial_{t} \alpha\right)\right)+\left\|\partial_{t}^{2} \alpha\right\|_{H^{2}}^{2} \leq 2\left\|\nabla \Delta \partial_{t} \alpha\right\|^{2}+C .
$$

Multiplying (1) by $-2 \Delta \partial_{t}^{2} u$, integrating over $\Omega$, we have

$$
\frac{d}{d t} E_{7}+2 \epsilon\left\|\partial_{t}^{2} u\right\|_{H^{1}}^{2} \leq C_{6}\left\|\partial_{t} u\right\|_{H^{1}}^{2}+\left\|\partial_{t}^{2} \alpha\right\|_{H^{1}}^{2}+C,
$$

where

$$
E_{7}=\left\|\partial_{t} u\right\|_{H^{1}}^{2}+2\left(\Delta u, \Delta \partial_{t} u\right)-2\left(f(u), \Delta \partial_{t} u\right)+2\left(\partial_{t} \alpha, \Delta \partial_{t} u\right) .
$$

Add (38), (46), (47), $\gamma_{11}(48), \gamma_{12}(49)$ and $\gamma_{13}(50)$ where $\gamma_{11}, \gamma_{12}$ and $\gamma_{13}>0$ are such that 


$$
\begin{gathered}
C_{1}-\gamma_{11}-C_{6} \gamma_{13}>0 ; \\
C_{4}-\gamma_{13}>0 ; \\
1-\gamma_{12}>0 ;
\end{gathered}
$$

we obtain

$$
\begin{aligned}
& \frac{d}{d t} E_{8}+C_{1}\left\|\partial_{t} u\right\|_{H^{1}}^{2}+C_{2}\left\|\partial_{t} u\right\|_{H^{2}}^{2}+C_{3}\|u\|_{H^{2}}^{2}+C_{4}\|\nabla \Delta u\|^{2}+C_{5}\|\alpha\|_{H^{2}}^{2}+C_{6}\|\nabla \Delta \alpha\|^{2} \\
& +C_{7}\left\|\partial_{t} \alpha\right\|_{H^{2}}^{2}+C_{8}\left\|\nabla \Delta \partial_{t} \alpha\right\|^{2}+C_{9}\left\|\partial_{t}^{2} \alpha\right\|_{H^{1}}^{2}+C_{10}\left\|\partial_{t}^{2} \alpha\right\|_{H^{2}}^{2}+C_{10} \epsilon\left\|\partial_{t}^{2} u\right\|_{H^{1}}^{2} \leq C,
\end{aligned}
$$

where

$$
\begin{aligned}
E_{8} & =E_{6}+\left(\epsilon\left\|\partial_{t} u\right\|_{H^{2}}^{2}+\|\nabla \Delta u\|^{2}+\left\|\partial_{t} \alpha\right\|_{H^{2}}^{2}+\|\nabla \Delta \alpha\|^{2}\right)+\left(2 \epsilon\left(\Delta \partial_{t} u, \Delta u\right)+\|u\|_{H^{2}}^{2}\right) \\
& +\gamma_{11}\left(2\left(\Delta \partial_{t} \alpha, \Delta \alpha\right)+\|\nabla \Delta \alpha\|^{2}\right)+\gamma_{12}\left(\left\|\nabla \Delta \partial_{t} \alpha\right\|^{2}+2\left(\nabla \Delta \alpha, \nabla \Delta \partial_{t} \alpha\right)\right) \\
& +\gamma_{13}\left(\left\|\partial_{t} u\right\|_{H^{1}}^{2}+2\left(\Delta u, \Delta \partial_{t} u\right)+2\left(f(u), \Delta \partial_{t} u\right)-2\left(\partial_{t} \alpha, \Delta \partial_{t} u\right)\right) .
\end{aligned}
$$

There exists $\beta>0$ independent of $\epsilon$ such that

$$
\beta E_{8} \leq C_{1}\left\|\partial_{t} u\right\|_{H^{1}}^{2}+C_{2}\left\|\partial_{t} u\right\|_{H^{2}}^{2}+C_{4}\|u\|_{H^{3}}^{2}+C_{6}\|\alpha\|_{H^{3}}^{2}+C_{8}\left\|\partial_{t} \alpha\right\|_{H^{3}}^{2} .
$$

Choosing $\gamma_{11}, \gamma_{12}, \gamma_{13}$ and $\gamma_{14}$ very small, there exists $C>0$ independent of $\epsilon$ such that

$$
C^{-1}\left(\left\|\zeta_{u}(t)\right\|_{\mathcal{E}^{3}(\epsilon)}^{2}+\left\|\partial_{t} \alpha(t)\right\|_{H^{3}}^{2}+\|\alpha(t)\|_{H^{3}}\right) \leq E_{8}(t) \leq C\left(\left\|\zeta_{u}(t)\right\|_{\mathcal{E}^{3}(\epsilon)}^{2}+\left\|\partial_{t} \alpha(t)\right\|_{H^{3}}^{2}+\|\alpha(t)\|_{H^{3}}\right) .
$$

Inserting estimate (52) into (51), we obtain

$$
\frac{d}{d t} E_{8}+\beta E_{8}+\left\|\partial_{t} u\right\|_{H^{1}}^{2}+\epsilon\left\|\partial_{t}^{2} u\right\|_{H^{1}}^{2}+\left\|\partial_{t}^{2} \alpha\right\|_{H^{1}}^{2} \leq C .
$$

Applying Gronwall's lemma to above estimate, thanks to estimate (53), we obtain the result.

Corollary 2. Assume assumptions of Theorem 3 hold and $0<\epsilon<1$. Then, the semigroup $S_{t}(\epsilon)$ associated with system (1) - (2) is dissipative in $\Phi_{3}$, i.e., it possesses a bounded absorbing set in $\Phi_{3}$.

This corollary is the straightforward consequence of above Theorem.

Theorem 6. Assume assumptions of Theorem 3 hold and $0<\epsilon<1$. Then, the semigroup $S_{t}(\epsilon)$ associated with system (1) - (2) possesses a global attractor $\mathcal{A}_{\epsilon}$ which is compact in $\Phi_{2}$, bounded in $\Phi_{3}$ and connected.

Proof. we already proved existence of bounded absorbed set $\mathcal{B}_{R_{0}}^{1}(\epsilon)$ in $\Phi_{2}$; it remains to split the solution $(u, \alpha) \in$ $B_{R_{0}}^{1}(\epsilon)$ as following

$$
(u, \alpha)=(v, \eta)+(\omega, \xi)
$$

such that semigroup $S_{t}(\epsilon)$ can be written as $S_{t}(\epsilon)=S_{t}^{1}(\epsilon)+S_{t}^{2}(\epsilon)$ with

$$
\begin{gathered}
S_{t}^{1}(\epsilon)\left(u(0), \partial_{t} u(0), \alpha(0), \partial_{t} \alpha(0)\right)=\left(v(t), \partial_{t} v(t), \eta(t), \partial_{t} \eta(t)\right), \\
S_{t}^{2}(\epsilon)(0,0,0,0)=\left(\omega(t), \partial_{t} \omega(t), \xi(t), \partial_{t} \xi(t)\right),
\end{gathered}
$$

where $S_{t}^{1}(\epsilon)$ is resolving operator of following linear hyperbolic system

$$
\begin{aligned}
\epsilon \partial_{t}^{2} v+\partial_{t} v-\Delta v & =\partial_{t} \eta \\
\partial_{t}^{2} \eta-\partial_{t} \Delta \eta-\Delta \eta & =-\partial_{t} v \\
v=\eta & =0 \text { over } \partial \Omega,
\end{aligned}
$$

$S_{t}^{2}(\epsilon)$ is resolving operator of following nonlinear hyperbolic system

$$
\begin{aligned}
\epsilon \partial_{t}^{2} \omega+\partial_{t} \omega-\Delta \omega+f(u) & =\partial_{t} \xi \\
\partial_{t}^{2} \xi-\partial_{t} \Delta \xi-\Delta \xi & =-\partial_{t} \omega \\
\omega=\xi & =0 \text { over } \partial \Omega
\end{aligned}
$$


and to show that $S_{t}^{1}(\epsilon)$ uniformly converges to 0 over all bounded set of $\Phi_{2}$ and $S_{t}^{2}(\epsilon)$ is regularizing in $\Phi_{3}$, when $t$ tends to $+\infty$.

We first prove that the semigroup of operator $S_{t}^{1}(\epsilon)$ uniformly converges to 0 in $\Phi_{2}$, when $t$ tends $+\infty$.

Multiplying (54) by $-2 \Delta \partial_{t} v$ and (55) by $-2 \Delta \partial_{t} \eta$, integrating over $\Omega$ and adding resulting equations, we have

$$
\frac{d}{d t}\left(\epsilon\left\|\partial_{t} v\right\|_{H^{1}}^{2}+\|v\|_{H^{2}}^{2}+\left\|\partial_{t} \eta\right\|_{H^{1}}^{2}+\|\eta\|_{H^{2}}^{2}\right)+2\left\|\partial_{t} v\right\|_{H^{1}}^{2}+2\left\|\partial_{t} \eta\right\|_{H^{2}}^{2}=0 .
$$

Multiply (54) by $-2 \Delta v$ and (55) by $-2 \Delta \eta$ and integrate over $\Omega$. We obtain

$$
\begin{aligned}
\frac{d}{d t}\left(\|v\|_{H^{1}}^{2}+2 \epsilon\left(\nabla v, \nabla \partial_{t} v\right)\right)+\|v\|_{H^{2}}^{2} & \leq C_{1}\left\|\partial_{t} \eta\right\|_{H^{2}}^{2}+2\left\|\partial_{t} v\right\|_{H^{1}}^{2} \\
\frac{d}{d t}\left(\|\eta\|_{H^{2}}^{2}+2\left(\nabla \eta, \nabla \partial_{t} \eta\right)\right)+\|\eta\|_{H^{2}}^{2} & \leq C_{2}\left\|\partial_{t} v\right\|_{H^{1}}^{2}+C_{3}\left\|\partial_{t} \eta\right\|_{H^{2}}^{2} .
\end{aligned}
$$

Multiply (54) by $2 \partial_{t}^{2} v$ and (55) by $-2 \Delta \partial_{t}^{2} \eta$, and integrate over $\Omega$. We have

$$
\begin{aligned}
\frac{d}{d t}\left(\left\|\partial_{t} v\right\|^{2}+2\left(\nabla v, \nabla \partial_{t} v\right)\right)+\epsilon\left\|\partial_{t}^{2} v\right\|^{2} & \leq C_{4}\left\|\partial_{t} \eta\right\|_{H^{2}}^{2}+2\left\|\partial_{t} v\right\|_{H^{1}}^{2} \\
\frac{d}{d t}\left(\left\|\partial_{t} \eta\right\|_{H^{2}}^{2}+2\left(\Delta \eta, \Delta \partial_{t} \eta\right)\right)+\left\|\partial_{t}^{2} \eta\right\|_{H^{1}}^{2} & \leq\left\|\partial_{t} v\right\|_{H^{1}}^{2}+2\left\|\partial_{t} \eta\right\|_{H^{2}}^{2} .
\end{aligned}
$$

Adding (58), $\gamma_{14}(59), \gamma_{15}(60), \gamma_{16}(61)$ and $\gamma_{17}(62)$ where $\gamma_{14}, \gamma_{15}, \gamma_{16}$ and $\gamma_{17}>0$ such that

$$
\begin{gathered}
1-2 \epsilon \gamma_{14}-C_{2} \gamma_{15}-2 \gamma_{16}-\gamma_{17}>0 \\
1-C_{1} \gamma_{14}-C_{3} \gamma_{15}-C_{4} \gamma_{16}-2 \gamma_{17}>0
\end{gathered}
$$

we have

$$
\frac{d}{d t} E_{9}+\|v\|_{H^{2}}^{2}+C_{1}\left\|\partial_{t} v\right\|_{H^{1}}^{2}+\|\eta\|_{H^{2}}^{2}+\left\|\partial_{t} \eta\right\|_{H^{2}}^{2}+\left\|\partial_{t}^{2} v\right\|_{H^{1}}^{2}+\left\|\partial_{t}^{2} \eta\right\|_{H^{1}}^{2} \leq 0
$$

where $C_{1}>0$ and

$$
\begin{aligned}
E_{9} & =\epsilon\left\|\partial_{t} v\right\|_{H^{1}}^{2}+\|v\|_{H^{2}}^{2}+\left\|\partial_{t} \eta\right\|_{H^{1}}^{2}+\|\eta\|_{H^{2}}^{2}+\gamma_{14}\left(\|v\|_{H^{1}}^{2}+2 \epsilon\left(\nabla v, \nabla \partial_{t} v\right)\right) \\
& +\gamma_{15}\left(\|\eta\|_{H^{2}}^{2}+2\left(\nabla \eta, \nabla \partial_{t} \eta\right)\right)+\gamma_{16}\left(\left\|\partial_{t} v\right\|^{2}+2\left(\nabla v, \nabla \partial_{t} v\right)\right) \\
& +\gamma_{17}\left(\left\|\partial_{t} \eta\right\|_{H^{2}}^{2}+2\left(\Delta \eta, \Delta \partial_{t} \eta\right)\right) .
\end{aligned}
$$

Moreover, for $\gamma_{15}, \gamma_{16}$ and $\gamma_{17}$ sufficiently small, there exists $\beta$ and $C>0$ such that

$$
\begin{aligned}
& \beta E_{9}(t) \leq\|v(t)\|_{H^{2}}^{2}+C_{1}\left\|\partial_{t} v(t)\right\|_{H^{1}}^{2}+\|\eta(t)\|_{H^{2}}^{2}+\left\|\partial_{t} \eta(t)\right\|_{H^{2}}^{2} \\
& C^{-1}\left\|\left(v(t), \partial_{t} v(t), \eta(t), \partial_{t} \eta(t)\right)\right\|_{\Phi_{2}}^{2} \leq E_{9}(t) \leq C\left\|\left(v(t), \partial_{t} v(t), \eta(t), \partial_{t} \eta(t)\right)\right\|_{\Phi_{2}}^{2} .
\end{aligned}
$$

Thanks to (64), estimate (63) can be written of the following form

$$
\frac{d}{d t} E_{9}+\beta E_{9}+\left\|\partial_{t}^{2} v\right\|^{2}+\left\|\partial_{t}^{2} \eta\right\|_{H^{1}}^{2} \leq 0
$$

Applying Gronwall's lemma to above estimate, thanks to estimate (65), we find

$$
\begin{aligned}
& \mathbb{D}[u](t)+\left\|\left(v(t), \partial_{t} v(t), \eta(t), \partial_{t} \eta(t)\right)\right\|_{\Phi_{2}}^{2}+\int_{0}^{t}\left(\left\|\partial_{t}^{2} v(s)\right\|^{2}+\left\|\partial_{t}^{2} \eta(s)\right\|_{H^{1}}^{2}\right) e^{-\beta(t-s)} d s \\
& \leq Q\left(\mathbb{D}[u](0), \|\left(u(0)\left\|_{H^{3}},\right\| \partial_{t} u(0)\left\|_{H^{2}},\right\| \alpha(0)\left\|_{H^{3}},\right\| \partial_{t} \alpha(0) \|_{H^{3}}\right) e^{-\beta t} .\right.
\end{aligned}
$$

where positive constants $C$ and $\beta$ are independent of $\epsilon$. Therefore the semigroup of operators $S_{t}^{1}(\epsilon)$ uniformly converges to 0 when $t$ tends to $+\infty$. To end, we prove that the semigroup $S_{t}^{2}(\epsilon)$ is regularizing in $\Phi_{3}$.

Thanks to assumptions of function $f$ and the fact that $u$ is continuous on $[0, T]$ such that $-1<u(t, x)<1 \quad \forall(t, x) \in$ $[0, T] \times \Omega$, we have $f^{\prime}(u) \nabla u, f^{\prime \prime}(u)(\nabla u)^{2}$ and $f^{\prime}(u) \Delta u \in L^{2}\left(0, T ; L^{2}(\Omega)\right), \forall T>0$. 
Multiplying (56) by $2\left(-\Delta \partial_{t} \omega+\Delta^{2} \partial_{t} \omega\right)$ and (57) by $2\left(-\Delta \partial_{t} \xi+\Delta^{2} \partial_{t} \xi\right)$, integrating over $\Omega$ and adding two resulting equations, we have

$$
\begin{aligned}
\frac{d}{d t} E_{10}+\left\|\partial_{t} \omega\right\|_{H^{1}}^{2}+2\left\|\partial_{t} \xi\right\|_{H^{2}}^{2}+\left\|\partial_{t} \omega\right\|_{H^{2}}^{2}+2\left\|\nabla \Delta \partial_{t} \xi\right\|^{2} & \leq\left\|f^{\prime}(u) \nabla u\right\|^{2}+2\left\|f^{\prime \prime}(u)(\nabla u)^{2}\right\|^{2} \\
& +2\left\|f^{\prime}(u) \Delta u\right\|^{2},
\end{aligned}
$$

where

$$
E_{10}=\epsilon\left\|\partial_{t} \omega\right\|_{H^{1}}^{2}+\epsilon\left\|\partial_{t} \omega\right\|_{H^{2}}^{2}+\|\omega\|_{H^{2}}^{2}+\|\nabla \Delta \omega\|^{2}+\left\|\partial_{t} \xi\right\|_{H^{1}}^{2}+\left\|\partial_{t} \xi\right\|_{H^{2}}^{2}+\|\xi\|_{H^{2}}^{2}+\|\nabla \Delta \xi\|_{H^{3}}^{2} .
$$

Multiplying (57) by $2 \Delta^{2} \partial_{t}^{2} \xi$ and integrating over $\Omega$, we have, owing to continuous injections $H^{2}(\Omega) \subset L^{\infty}(\Omega)$ and $H^{3}(\Omega) \subset L^{\infty}(\Omega)$

$$
\frac{d}{d t}\left(\left\|\nabla \Delta \partial_{t} \xi\right\|^{2}+2\left(\nabla \Delta \xi, \nabla \Delta \partial_{t} \xi\right)\right)+\left\|\partial_{t}^{2} \xi\right\|_{H^{2}}^{2} \leq C .
$$

Summing (66) and $\gamma_{18}(67)$ where $\gamma_{18}>0$, we obtain

$$
\frac{d}{d t} E_{11} \leq C\left(\left\|f^{\prime}(u) \nabla u\right\|^{2}+\left\|f^{\prime}(u)(\nabla u)^{2}\right\|^{2}+2\left\|f^{\prime \prime}(u) \Delta u\right\|^{2}+1\right),
$$

where

$$
E_{11}=E_{10}+\gamma_{18}\left(\left\|\nabla \Delta \partial_{t} \xi\right\|^{2}+2\left(\nabla \Delta \xi, \nabla \Delta \partial_{t} \xi\right)\right)
$$

which implies, by integration between 0 and $t$

$$
E_{12}(t) \leq C\left(T^{2}+1\right) Q\left(\left\|u_{0}\right\|_{H^{2}},\left\|\partial_{t} u_{0}\right\|,\left\|\alpha_{0}\right\|_{H^{2}},\left\|\alpha_{1}\right\|_{H^{2}}\right) .
$$

For sufficiently small $\gamma_{18}>0$, there exists $C>0$ such that

$$
C\left\|\left(\omega(t), \partial_{t} \omega(t), \xi(t), \partial_{t} \xi(t)\right)\right\|_{\Phi_{3}}^{2} \leq E_{12}(t) .
$$

Thanks to above estimate, estimate (69) implies

$$
\left\|\left(\omega(t), \partial_{t} \omega(t), \xi(t), \partial_{t} \xi(t)\right)\right\|_{\Phi_{3}}^{2} \leq C\left(T^{2}+1\right) Q\left(\mathbb{D}[u](0),\left\|u_{0}\right\|_{H^{2}},\left\|u_{1}\right\|,\left\|\alpha_{0}\right\|_{H^{2}},\left\|\alpha_{1}\right\|_{H^{2}}\right),
$$

then, the semigroup $S_{t}^{2}(\epsilon)$ is regularizing on $\Phi_{3}$. This finishes the proof of the Theorem.

\section{Discussion}

In this article we have proven as in (Moukoko, 2014, 2015) that the Caginalp hyperbolic phase-field system with singular potential, has a unique solution and the global attractor. It remains to study the existence of exponential attractors and the robust family of exponential attractors for Caginalp hyperbolic phase-field system with singular potential.

We can also complete this work by studying Caginalp hyperbolic phase-field system with other types of boundary conditions and regular or singular potential.

\section{Acknowledgements}

The authors wish to thank Alain Miranville and Brice Landry Doumbé Bangola for many stimulating discussions. The authors are very grateful to referees for the care that they took in reading this article.

\section{References}

Caginalp, G. (1986). An analysis of a phase field model of a free boundary. Arch. Rational Mech. Anal., 92(3), 205-245. http://dx.doi.org/10.1007/bf00254827

Cherfils, L., Gatti, S., \& Miranville, A. (2008). Existence of global solutions to the Caginalp phase-field system with dynamic boundary conditions and singular potentials. J. Math. Anal. Appl., 348(2), 1029-1030.

Cherfils, L., \& Miranville, A. (2009). On the Caginalp system with dynamic boundary conditions and singular potentials. Appl. Math., 54(2), 89-115.

Conti, M., Gatti, S., \& Miranville, A. (2012). Asymptotic behavior of the Caginalp phase-field system with coupled dynamic boundary conditions. Discrete Contin. Dyn. Syst. Ser. S, 5(3), 485-505. 
Fabrie, P., Galusinski, C., Miranville, A., \& Zelik, S. (2004). Uniform exponential attractors for a singularly perturbed damped wave equation. Discrete Contin. Dyn. Syst., 10(1-2), 211-238.

Efendiev, M., Miranville, A., \& Zelik, S. (2003). Infinite dimensional exponential attractors for a non-autonomous reaction-diffusion system., Math. Nachr., 248/249(72-96).

Efendiev, M., Miranville, A., \& Zelik, S. (2004). Exponential attractors for a singularly perturbed Cahn-Hilliard system. Math. Nachr., 272, 11-31.

Grasselli, M., Miranville, A., Pata, V., \& Zelik, S. (2007). Well-posedness and long time behavior of parabolichyperbolic phase-fielf sistem with singular potentials. Math. Nachr., 280, 13-14.

Miranville, A., \& Quintanilla, R. (2009). A generalization of the Caginalp phase-field system based on the Cattaneo law. Nonlinear Anal.,71(5-6), 2278-2290.

Miranville, A., \& Quintanilla, R. (2009). Some generalizations of the Caginalp phase-field system. Appl. Anal., 88(6). http://dx.doi.org/10.1080/00036810903042182

Miranville, A., \& Zelik, S. (2008). Attractors for dissipative partial equations in bounded and unbounded domains, in Handbook of Differential Equations, Evolutinary Partial Differential Equations, Elsevier, Amsterdam.

Moukoko, D. (2014). Well-posedness and long time behavior of a hyperbolic Caginalp system phase-field sytem. JAAC, 4(2), 151-196.

Moukoko, D. (2015). Etude de modèles hyperboliques de champ de phases de Caginalp, Thèse Unique, Faculté des Sciences et Techniques, Université Marien Ngouabi.

\section{Copyrights}

Copyright for this article is retained by the author(s), with first publication rights granted to the journal.

This is an open-access article distributed under the terms and conditions of the Creative Commons Attribution license (http://creativecommons.org/licenses/by/3.0/). 